\title{
Simulation of Particle Segregation in Fluidized Beds
}

\author{
Janitha C. Bandara Rajan K. Thapa Britt M.E. Moldestad Marianne S. Eikeland \\ Process Energy and Environmental Technology, University College of Southeast Norway, Norway \\ \{Janitha.bandara, rajan.k.thapa, britt.moldestad, Marianne.Eikeland\}@usn.org
}

\begin{abstract}
Fluidization technology is widely used in solid processing industry due to the high efficiency, high heat and mass transfer rate and uniform operating conditions throughout the reactor. Biomass gasification is an emerging renewable energy technology where fluidized bed reactors are more popular compared to fixed bed reactor systems due to their scalability to deliver high throughput. Fluidization of large biomass particles is difficult, and the process is therefore assisted by a bed material with higher density. The combination of different types of particles makes it challenging to predict the fluid-dynamic behavior in the reactor. Computational particle fluid dynamics simulations using the commercial software Barracuda VR were performed to study the fluidization properties for a mixture of particles with different density and size. The density ratio for the two types of particles was six, which is the typical ratio for bed material to biomass in a gasifier. The results from simulations with Barracuda VR regarding bed pressure drop and the minimum fluidization velocity, show good agreement with available experimental data. The deviation between experimental data and simulations are less than $12 \%$. Particle segregation was clearly observed both in the simulations and in the experimental study.
\end{abstract}

Keywords: fluidization, particle density and size, pressure drop, minimum fluidization velocity, drag models, computational particle fluid dynamics

\section{Introduction}

Fluidized beds are extensively utilized in mechanical processes, energy technology and processing industries (Vollmari et al, 2016). The process was introduced in fluid catalytic cracking (FCC) in the petroleum industry as an alternative to thermal cracking (Horio, 2013; Winter and Schratzer, 2013).

In contrast to the fluid state reactions, solid state processing is rather challenging. Fluidization emerged as a remarkable technique due to high efficiency, low emissions and excellent heat and mass transfer characteristics (Winter and Schratzer, 2013). However, even with the knowledge over decades, the complex dynamics of the fluidization is not fully understood (Sánchez-Delgado et al, 2011).
Thermal conversion of biomass to energy is a renewable and deep-rooted process where fluidized bed combustors and gasifiers are attaining more attention compared to the fixed bed designs, due to the flexibility of scaling up along with uniform operating conditions throughout the reactor (Sau et al, 2007). A bed-material is commonly used as a fluidizing aid, because the biomass particles alone are more difficult to fluidize. Apart from assisting fluidization, the bed material can act either as a thermal energy carrier, a catalyst or both. Therefore, the presence of particles having different densities and sizes is a common in fluidized bed combustors and gasifiers since the bed material, biomass and char are fluidized together. This may lead to segregation where lighter biomass particles rise to the top of the bed while the heavier bed material moves to the bottom. However, it is said that the difference in density affects more than the difference in particle size on the tendency of segregation (Cooper and Coronella, 2005). Minimum fluidization velocity is another important parameter for gasification, because the system operates under restricted gas supply. If the mass flowrate relevant for the bubbling fluidization velocity is greater than the stoichiometric requirement for gasification, more fully oxidized products such as carbon dioxide $\left(\mathrm{CO}_{2}\right)$ could be present in the producer gas, which consequently reduces the producer gas quality. In such situations, it is necessary to bring down the minimum fluidization velocity by making necessary changes in the particle properties and the reactor geometry. Hence, it is important to study the effect of different particle mixtures on the solid flow pattern, the minimum fluidization velocity and the pressure drop behavior.

\section{Fluidization}

A collection of particles, such as a sandy beach, acts as a solid due to gravitational and surface forces. However, it is possible to change the solid like existence of a collection of particles by applying an upward fluid flow with a sufficient velocity to counter-weight the prior mentioned forces with the drag force. Once the static forces between the particles are overcome and all the forces are in perfect balance, the particles starts behaving like a fluid, which is known as fluidization. The minimum superficial gas velocity at which the 
transition from fixed to fluidized bed occurs is referred as the minimum fluidization velocity (Horio, 2013).

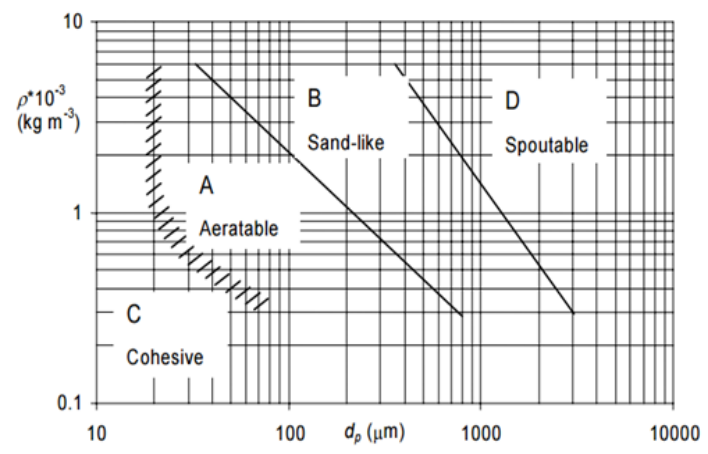

Figure 1. Geldart powder classification

Size, shape and density of the particles together with the fluid superficial velocity, characterize the behavior of fluidization where homogeneous, bubbling, slugging, spouting and turbulent are the well-known regimes. Figure 1 represents Geldart's classification of particles into the four different regimes A, B, C and D (Geldart, 1973; Fotovat et al, 2015).

According to the Geldart, type " $\mathrm{A}$ " particles are small with typical density is less than $1400 \mathrm{~kg} / \mathrm{m}^{3}$. The bed expands at the minimum fluidization velocity and the regime changes to bubbling fluidized bed as the gas velocity increases. Most of the type B materials are in the range of $40 \mu \mathrm{m}$ to $500 \mu \mathrm{m}$ in size with density ranging from $1400 \mathrm{~kg} / \mathrm{m}^{3}$ to $4000 \mathrm{~kg} / \mathrm{m}^{3}$. The bubbling regime starts as soon as the minimum fluidization velocity is reached. Due to fine particles and strong cohesive forces, group $\mathrm{C}$ powders show high resistance against fluidization. Particles with large mean size are classified as group $\mathrm{D}$, which normally operates at the spouting regime. At high superficial gas velocities, all the four types of powders obtain fast and turbulent fluidization, and at further increase in the gas velocity, pneumatic transport will occur.

Pressure drop, minimum fluidization velocity and solid flow patterns are important parameters for design, optimization and operation of fluidized beds. In bubbling fluidization, the bubble activity is the main factor, which influences on the solid flow pattern inside the reactor. Particles are carried up inside the bubble, which is referred to as the bubble wake. These particles are brought to the surface of the bed, which leads to mixing of the top and bottom fractions (Sau et al., 2007). As bubbles move upwards, the void leaving behind immediately below the bubble is filled with surrounding particles. Whenever multiple particle species are involved, heavier particles tend to accumulate at the bottom and lighter at the bed surface, which are referred as 'jetsam' and 'flotsam' respectively (Cooper and Coronella, 2005). Solid flow patterns affect the characteristics such as rate of heat and mass transfer, reaction intensity, particle attrition, mixing, segregation and internal corrosion. In addition to the particle and gas properties, bed geometry, aspect ratio and gas distribution make a considerable influence on the fluidization parameters.

Experimental investigations at industrial scale fluidization involve high costs, while it is not easy to scale up from lab scale to industrial scale. Hence, detailed analysis of the fluidization process via simulations provides a better understanding at reduced costs and time (Vollmari et al, 2016).

Computational Fluid Dynamics (CFD) has become a powerful tool, and different software such as Barracuda VR, FLUENT, OpenFOAM and MFIX have been developed for CFD simulations. The objective of this paper is to analyze the effect of the presence of particles with different densities and sizes in the fluidized bed, on the pressure drop and minimum fluidization velocity, using the commercial CPFD software Barracuda VR. However, as some correlations used in CFD packages are empirical or semi-empirical, it is important to validate the results against experimental data performed on a fluidized bed with the same size and design (Taghipour et al, 2005). Hence, the simulation results are validated against data from experimental studies performed by Thapa et al (2011).

\section{Experimental Setup}

Thapa et al. (2011) have carried out experimental investigations of fluidization using particles with different densities and sizes. The experimental rig was an $84 \mathrm{~mm}$ diameter cylindrical column with a homogeneous air distributor at the bottom. Nine pressure measuring points have been used which were equally spaced with $100 \mathrm{~mm}$ along its height. Figure 2 illustrates the arrangements of the experimental rig.

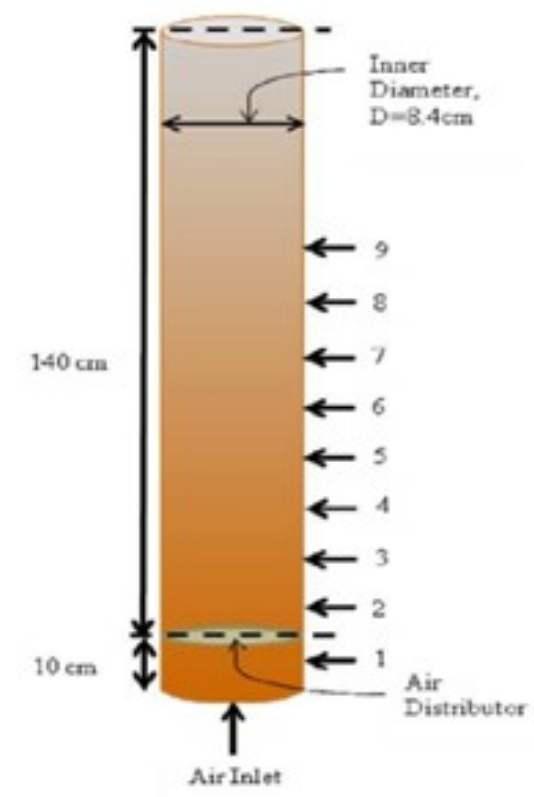

Figure 2. Geldart powder classification 
In a real gasification system, the densities of the bed material and char/wood particles are approximately $2500 \mathrm{~kg} / \mathrm{m}^{3}$ and $400-500 \mathrm{~kg} / \mathrm{m}^{3}$ respectively. Here, $\mathrm{ZrO}_{2}$ and plastic beads were used to represent the bed material and biomass respectively, keeping the density ratio at a value of six, which is about the same as for a real gasification system.

\section{Simulation Setup}

Two fluid model (TFM) and Discrete particle/element model (DPM/DEM) are the two basic approaches, which are also referred to as the Eulerian-Eulerian and the Eulerian-Lagrangian models respectively. TFM considers that both the fluid and the particle phases are fully interpenetrating continua, where the solid phase properties are calculated based on the kinetic theory of granular flow (Jayarathna, 2014).

In the DPM simulations, the fluid phase is treated as a continuous medium whereas the particles are considered as individual components. Using DPM, the equations are solved for each particle. This approach needs the collision models to address the non-ideal interaction between particles. However, the DPM approach is computationally expensive, and it is only possible to handle a limited number of particles.

Computational particle fluid dynamics (CPFD) is an extension of CFD, which facilitates the multi-phase systems such as fluidization. Barracuda VR is specially developed for multiphase CPFD simulations, which utilizes the theory of multiphase Particle in Cell (MP PIC). In the PIC approach, a collection of real particles, which is referred as a 'computational particle', is performed in the Lagrangian particle modeling while the fluid phase is treated as a continuum (Andrews and O'Rourke, 1996).

The computational setup is presented in Figure 3. Air exit at the top of the column was set as a pressure boundary with atmospheric conditions and with no particle exit. Stepwise increase of the air velocity at the inlet flow boundary (bottom of the geometry) was performed in order to calculate the minimum fluidization velocity. Both pressure and temperature were defined at the inlet flow boundary, which allows the system to calculate the mass flow rate of the gas. However, this inlet pressure applies only for the initial time step and the system itself calculates the required pressure according to the bed pressure drop. The time step was set to 0.001 seconds for the simulations and whenever the user defined time step is too high, Barracuda VR re-calculates it. This guarantees the stability of the simulation.

Simulations were carried out for both the individual particle types and for mixtures of the two types of particles. The effect on the minimum fluidization velocity was observed. Table 1 summarizes the rest of the simulation parameters.

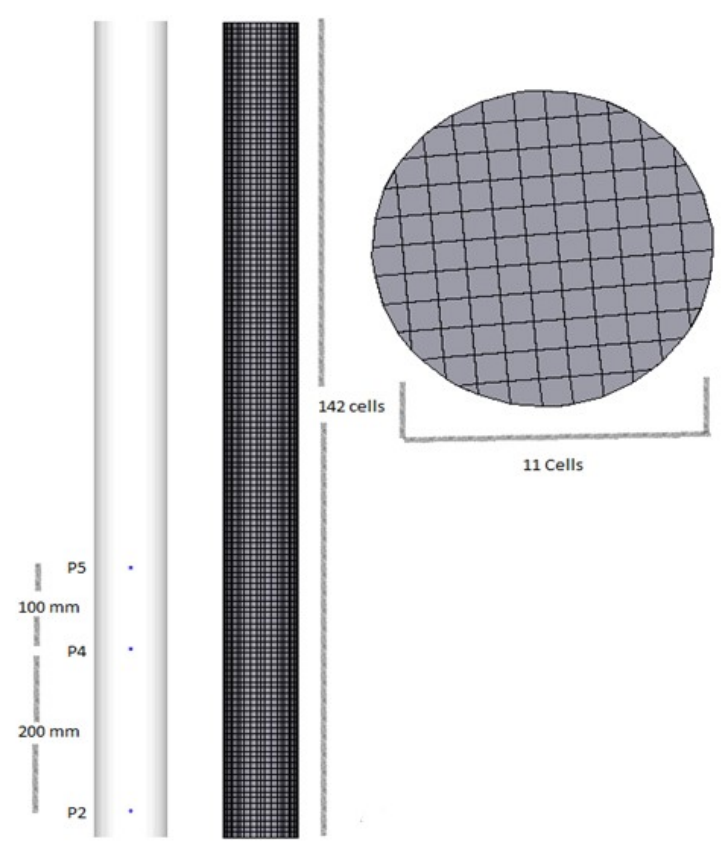

Figure 3. Computational grid for the simulation and pressure transient data points

As illustrated in Figure 3, the pressure monitor points P2, P4 and P5 were included. Uniform meshing and 17061 cells were used for all the simulations. The system was simulated for 5 seconds at each velocity step and the pressure, averaged over the last second of each time step, was used in the data analysis. The pressure drop was calculated as the pressure drop per unit height $(\mathrm{mbar} / \mathrm{m})$ in order to minimize the errors. The pressure drop gradient as a function of the superficial gas velocity for both experimental data and simulation results, were plotted in the same chart to compare the results.

Table 1. Simulation parameters

\begin{tabular}{ll}
\hline Parameter & Value \\
\hline $\mathrm{ZrO}_{2}$ density & $5850 \mathrm{~kg} / \mathrm{m}^{3}$ \\
\hline Plastic density & $964 \mathrm{~kg} / \mathrm{m}^{3}$ \\
\hline $\mathrm{ZrO}_{2}$ particle size & $709 \mu \mathrm{m}$ \\
\hline Plastic particle size & $3500 \mu \mathrm{m}$ \\
\hline Bed height & $340 \mathrm{~mm}$ \\
\hline Bed width & $84 \mathrm{~mm}$ \\
\hline Temperature & $300 \mathrm{~K}$ \\
\hline Sphericity & 1 \\
\hline Maximum packing & 0.6 \\
\hline $\begin{array}{l}\text { Max. Momentum redirection from } \\
\text { collisions }\end{array}$ & $40 \%$ \\
\hline Normal to wall momentum retention & 0.3 \\
\hline Tangent to wall momentum retention & 0.99 \\
\hline Stress model parameters & Default \\
\hline
\end{tabular}




\section{Results and Discussion}

A real gasification/combustion system is a reaction volume with non-isothermal conditions with a wide range of particle sizes, shapes and densities. A fluidized bed gasifier includes wood chips $(1-5 \mathrm{~cm})$, reacted char (larger particle range) and bed material (400 to 600 microns). Large plastics beads with a wide shape distribution are used in experiments and simulations providing a good approximation to the wood particles. A density ratio of six between the two particle types is used to get a good agreement between the cold flow model and the actual gasification model. The use of air at ambient temperature in contrast to the actual system of high temperature air or steam is another challenge when predicting the real world system. However, cold bed data may provide an overview to get a good understanding of fluidization of particle mixtures having different densities and particle sizes. The study is an initial step in simulation of a complex fluidized bed gasification process.

\subsection{Minimum Fluidization Velocity and Bed Pressure Drop}

The simulations were carried out for pure $\mathrm{ZrO}_{2}$ particles, pure plastic beads and with mixtures of $10 \%$ and $20 \%$ plastic beads with $\mathrm{ZrO}_{2}$ as the rest. The pressure drop gradient over the bed height was calculated using equation (1).

$$
\frac{\Delta P}{H}=\frac{P_{4}-P_{2}}{200}
$$

The minimum fluidization velocity is determined as the velocity where the increasing pressure drop starts to drop creating a maximum. However, the minimum fluidization velocity lies slightly below that value due to initial packed bed resistance. Hence, the best way to determine the minimum fluidization velocity is to carry out the experiments from high velocities to lower velocities as well, and get the average values for the two cases. Wen-Yu, Ergun drag model was used in the Barracuda VR simulations and it is available as an inbuilt model.

Experimental data for the pure plastic beads and simulation results based on the Wen-Yu-Ergun drag model is illustrated in Figure 4. The Wen-Yu-Ergun model is a combined model for the drag force, which was proposed by Gidaspow. The combined model selects the interface momentum transfer coefficient $\left(\mathrm{K}_{\mathrm{sg}}\right)$ by either Wen-Yu or Ergun depending upon the bed void fraction. If the gas volume fraction is greater than 0.8 , the Wen-Yu correlation is selected. The Wen$\mathrm{Yu}$ correlation is expressed in equation (2).

$$
K_{S g}=\frac{3}{4} \frac{C_{D} \rho_{g} \varepsilon_{g}\left(1-\varepsilon_{g}\right)\left(u_{s}-u_{g}\right)}{d_{p}} \varepsilon_{g}^{-2.65}
$$

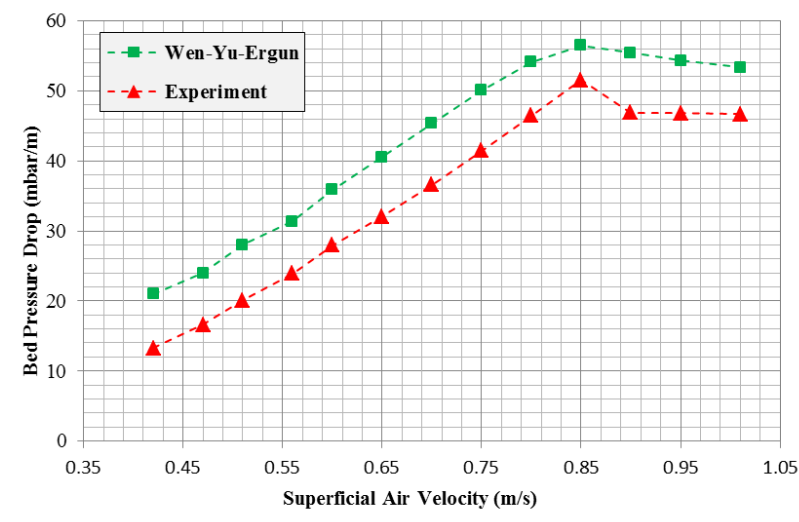

Figure 4. Simulation and experimental data for plastic beads

Where $\mathrm{CD}$ is given by:

$$
C_{D}=\frac{24}{\varepsilon_{g} R e_{s}}\left[1+0.15\left(\varepsilon_{g} R e_{s}\right)^{0.687}\right]
$$

Otherwise, when the void fraction is lower than 0.8 , the Ergun correlation is selected as in equation (4):

$$
K_{s g}=150 \frac{\mu_{g}\left(1-\varepsilon_{g}\right)^{2}}{\varphi^{2} d_{p}^{2} \varepsilon_{g}}+1.75 \frac{\rho_{g}\left(u_{g}-u_{s}\right)\left(1-\varepsilon_{g}\right)}{\varphi d_{p}}
$$

Where $u_{g}$ is the superficial gas velocity, $\mu_{\mathrm{g}}$ is the gas viscosity, $\rho_{\mathrm{g}}$ is the density of the gas, $\rho_{\mathrm{s}}$ is the density of particles, $d_{p}$ is the mean particle diameter, $\varphi$ is the particle sphericity, $\mathrm{Re}_{\mathrm{s}}$ is the particle Reynolds number and $\varepsilon_{\mathrm{g}}$ is the volume fraction of the gas.

Experimental results for plastic beads show that the minimum fluidization velocity and maximum bed pressure drop are $0.85 \mathrm{~m} / \mathrm{s}$ and $50 \mathrm{mbar} / \mathrm{m}$ respectively. The pressure drop from the simulations is higher compared to the experimental data. The pressure drop gradient at minimum fluidization velocity is measured to be $52 \mathrm{mbar} / \mathrm{m}$ in the experiments and calculated as 56 $\mathrm{mbar} / \mathrm{m}$ in the simulations. This deviation are mainly due to the uncertainty of bed void fraction data, particle sphericity and particle size distribution. Bed voidage is a strong function of particle size distribution and sphericity. Simulations with increased void fraction illustrated that the bed pressure drop reached the experimental values without changing the minimum fluidization velocity. Further, the pressure tapping points are at the wall of the experimental rig and in the real situation, there is a tendency of air to escape through loosely packed regions near the wall.

However, the minimum fluidization velocity is the same for the experiments and the simulations, having a value of $0.85 \mathrm{~m} / \mathrm{s}$. The simulation results for the bed pressure drop is higher than the experimental data also after the fluidization regime is reached. Parameters of the particle stress model affect considerably in the fluidization regime while it has a negligible effect in the packed bed region. As the main objective for this study 
was to analyze the minimum fluidization velocity and particle segregation, default values of the particle stress model were used. This can be a reason for the deviation in pressure drop in the fluidization regime.

The behavior of pure $\mathrm{ZrO}_{2}$ particles is plotted in Figure 5. The pressure drop from the simulations is slightly higher than the experimental data. The simulated bed pressure drop at minimum fluidization is $350 \mathrm{mbar} / \mathrm{m}$, which gives a deviation of about $6 \%$ compared to the experimental data of $330 \mathrm{mbar} / \mathrm{m}$. Minimum fluidization velocities are $0.67 \mathrm{~m} / \mathrm{s}$ and $0.7 \mathrm{~m} / \mathrm{s}$ in the experiment and simulation respectively. The particle size distribution is an important factor for both the minimum fluidization velocity and the bed pressure drop. When the particle size distribution is wide, the void fraction in the bed becomes lower. However, the particle size distribution for $\mathrm{ZrO}_{2}$ in the experimental study has not been reported, and only the mean diameter of $709 \mu \mathrm{m}$ was available. Therefore, using uniform size particles could be one of the reasons for the deviation in the results.

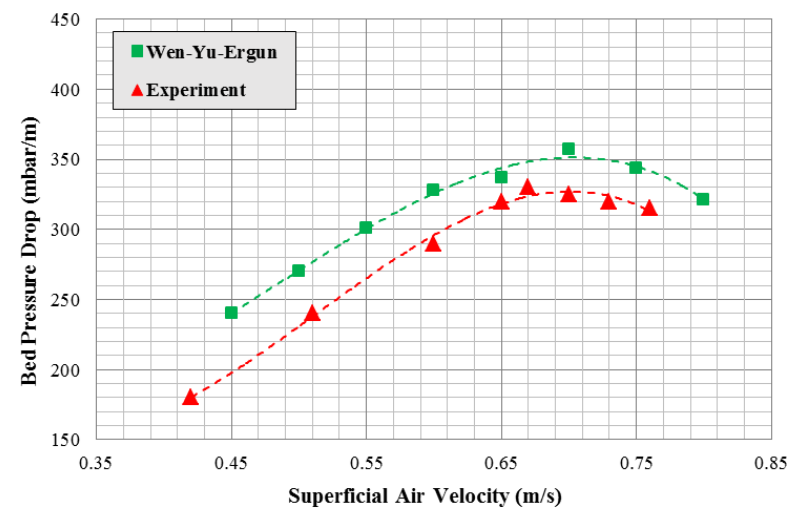

Figure 5. Simulation and experimental data for $\mathrm{ZrO}_{2}$

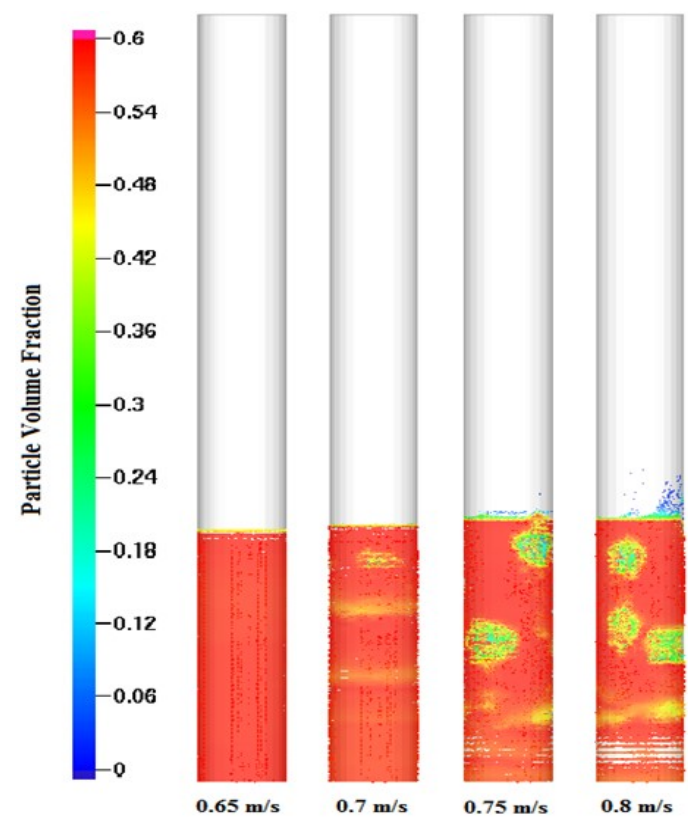

Figure 6. Transition from fixed to fluidized bed of $\mathrm{ZrO}_{2}$
A graphical display of transition from fixed bed to fluidized bed followed by bubble formation for $\mathrm{ZrO} 2$ particles is captured in Figure 6. There was a high tendency of bubble formation and bubble rise near the walls.

The simulation results for pure particles of plastic and $\mathrm{ZrO}_{2}$ agree well with the experimental results. Simulations using different drag models were performed, and Wen-Yu-Ergun was found to have the best agreement with the experimental data. Therefore, simulations for particle mixtures of plastic and $\mathrm{ZrO}_{2}$ were performed with this drag model. Figure 7 and Figure 8 depict the superficial air velocity versus bed pressure drop for particle mixtures of $10 \%$ and $20 \%$ plastic beads respectively.

The simulation results of both the mixtures show similar behaviors. The bed pressure drop is higher than the experimental data in the fixed bed regime, while the gradient sharply drops down close to the minimum fluidization velocity. In contrast to the case of pure particles, it is difficult to observe a sharp change in pressure drop gradient at the state of minimum fluidization. Typically, when two particles with different minimum fluidization velocities are mixed, local fluidization behavior of one component (with lower minimum fluidization velocity) can be observed before the total bed starts to fluidize. This phenomenon breaks the linear relationship of air velocity to pressure drop in the fixed bed regime.

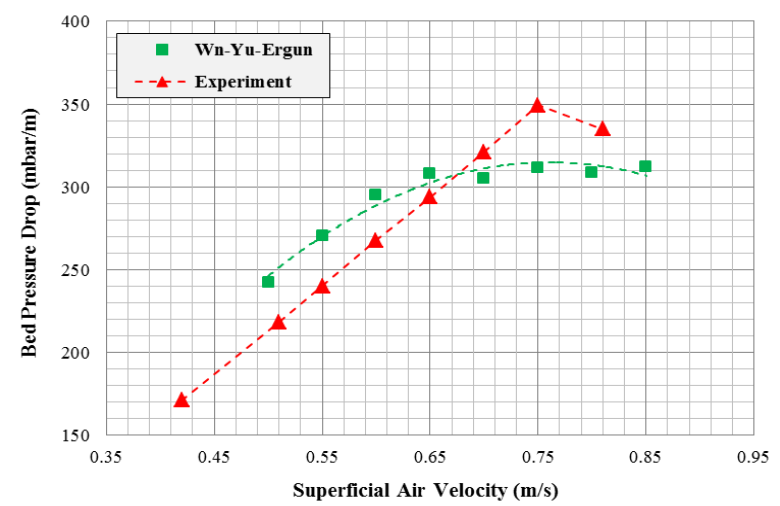

Figure 7. Simulation results $10 \%$ plastic and $\mathrm{ZrO}_{2}$

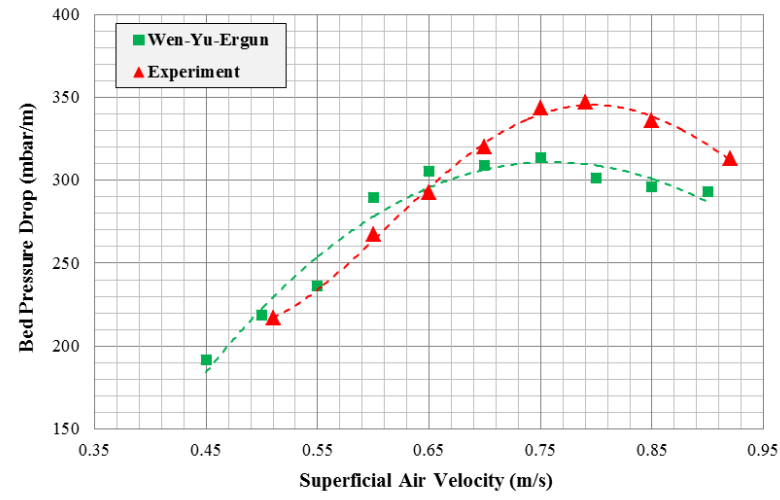

Figure 8. Simulation results $20 \%$ plastic and $\mathrm{ZrO}_{2}$ 
In the simulations, the pressure drop tends to be about constant when the gas velocity changes from 0.65 to $0.75 \mathrm{~m} / \mathrm{s}$. After reaching the minimum fluidization velocity, the pressure gradient starts to decrease. Both mixtures have high fraction of $\mathrm{ZrO}_{2}$ and that could be one reason for the steady pressure drop when the velocity exceed $0.65 \mathrm{~m} / \mathrm{s}$, which is approximately the minimum fluidization velocity of $\mathrm{ZrO}_{2}$. The experimental minimum fluidization velocities were 0.75 $\mathrm{m} / \mathrm{s}$ and $0.77 \mathrm{~m} / \mathrm{s}$ for the mixtures with $10 \%$ and $20 \%$ plastic particles, respectively. In the simulations, the minimum fluidization velocity was $0.75 \mathrm{~m} / \mathrm{s}$ for both the mixtures. It is rather difficult to pick a sharp turning point for minimum fluidization from the simulation results. However, the simulation result follows the trend of the experimental data.

\subsection{Particle Segregation}

The distribution of $\mathrm{ZrO}_{2}$ particles and plastic beads in the bed as a function of time is given in Figure 9. The figure clearly depicts that the lower density particles moves upward while the higher density fraction accumulates at the bottom. Even though plastic beads have significantly higher particle size compared to $\mathrm{ZrO}_{2}$, the lower density makes it move up to the surface. Therefore, it is vital to know the time taken for complete segregation. Once the low density particles have moved to the bed surface, the bed properties are changed from the initial conditions and this may lead to fluidization of only one of the segregated layers. The segregation of particles was also observed in the experimental study of Thapa et al (2011).

When simulating mixtures with different particle sizes in Barracuda VR, it is recommended to activate the blended acceleration model (BAM) which averages the different velocities caused by the size differences.

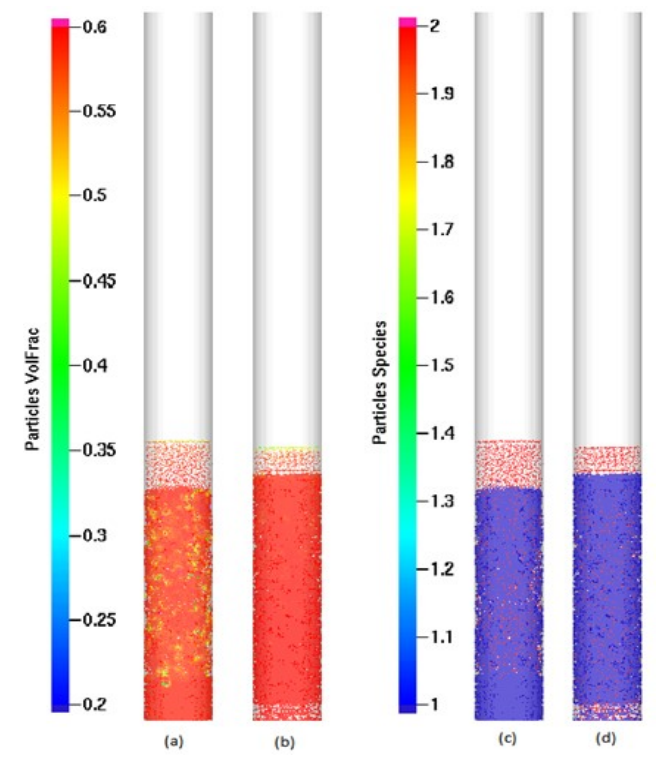

Figure 9. Particle segregation of a mixture of $\mathrm{ZrO}_{2}$ and $10 \%$ plastic beads, a) and c) $15 \mathrm{~s}$ after fluidization, b) and d) $5 \mathrm{~s}$ after fluidization

\section{Conclusions}

The results from simulations with Barracuda VR show good agreement with available experimental data. The deviation between experimental data and simulations are less than $12 \%$ for both the bed pressure drop and the minimum fluidization velocity.

The selection of drag model is crucial for the simulation results, and therefore the model should be carefully chosen. However, it is challenging to predict the suitability of a particular drag model for a particular system and it is recommended to get the model validated against experimental data. The deviation between experimental data and simulations were less than $12 \%$. Insufficient information about particle size distribution for the $\mathrm{ZrO}_{2}$, together with uncertainty about particle sphericity and the particle volume fraction can influence on the simulation results. The large size of plastic beads was a constraint in order to use a fine mesh in the simulations.

The users can define their own drag models in Barracuda VR, and are allowed to change the particle normal stress model parameters. However, the system can be further optimized by changing the drag model, the particle stress model parameters and the wall interaction coefficients.

Particle separation was clearly observed and it should be further analyzed with different air velocities and with different mixtures of particles. More analysis is possible with varied particle size to get the optimized size ratio for the particle mixtures to avoid segregation.

\section{Acknowledgements}

The authors like to thank Mr. Chameera Jayarathna and Mr. Amila Chandra for their support in handling Barracuda VR and SolidWorks.

\section{References}

M.J. Andrews and P. J. O'Rourke . The multiphase particlein-cell (MP-PIC) method for dense particulate flows. International Journal of Multiphase Flow, 22(2): 379-402, 1996.

S. Cooper and C. J. Coronella. CFD simulations of particle mixing in a binary fluidized bed. Powder Technology, 151(1): 27-36, 2005.

F. Fotovat, R. Ansart, M. Hemati, O. Simonin and J. Chaouki. Sand-assisted fluidization of large cylindrical and spherical biomass particles: Experiments and simulation. Chemical Engineering Science, 126(Supplement C): 543-559, 2015.

D. Geldart. Types of gas fluidization. Powder Technology, 7(5): 285-292, 1973.

M. Horio. 1 - Overview of fluidization science and fluidized bed technologies A2 - Scala, Fabrizio. Fluidized Bed Technologies for Near-Zero Emission Combustion and Gasification, Woodhead Publishing: 3-41, 2013. 
C.K. Jayarathna, B. M. Halvorsen and L.-A. Tokheim. Experimental and Theoretical Study of Minimum Fluidization Velocity and Void Fraction of a Limestone Based $\mathrm{CO}_{2}$ Sorbent. Energy Procedia, 63(Supplement C): 1432-1445, 2014

S. Sánchez-Delgado, J. A. Almendros-Ibáñez, N. GarcíaHernando and D. Santana. On the minimum fluidization velocity in 2D fluidized beds. Powder Technology, 207(1): 145-153, 2011.

D.C. Sau, S. Mohanty and K. C. Biswal. Minimum fluidization velocities and maximum bed pressure drops for gas-solid tapered fluidized beds. Chemical Engineering Journal, 132(1): 151-157, 2007.

F. Taghipour, N. Ellis and C. Wong. Experimental and computational study of gas-solid fluidized bed hydrodynamics. Chemical Engineering Science, 60(24): 6857-6867, 2005.

R.K. Thapa, C. Rautenbach and B. M. Halvorsen. Investigation of flow behavior in biomass gasifier using electrical capacitance tomography (ECT) and pressure sensors. International Conference on Polygeneration Strategies. F. M. Hofbauer. Vienna University of Technology, Austrian National Library: 97-106, 2011.

K. Vollmari, R. Jasevičius and H. Kruggel-Emden. Experimental and numerical study of fluidization and pressure drop of spherical and non-spherical particles in a model scale fluidized bed. Powder Technology, 291(Supplement C): 506-521, 2016.

F. Winter and B. Schratzer. 23 - Applications of fluidized bed technology in processes other than combustion and gasification A2 - Scala, Fabrizio. Fluidized Bed Technologies for Near-Zero Emission Combustion and Gasification, Woodhead Publishing: 1005-1033, 2013. 